\title{
THE HOMOTOPY TYPES OF MOMENT-ANGLE COMPLEXES FOR FLAG COMPLEXES
}

\author{
JELENA GRBIĆ, TARAS PANOV, STEPHEN THERIAULT, AND JIE WU
}

\begin{abstract}
We study the homotopy types of moment-angle complexes, or equivalently, of complements of coordinate subspace arrangements. The overall aim is to identify the simplicial complexes $K$ for which the corresponding moment-angle complex $\mathcal{Z}_{K}$ has the homotopy type of a wedge of spheres or a connected sum of sphere products. When $K$ is flag, we identify in algebraic and combinatorial terms those $K$ for which $\mathcal{Z}_{K}$ is homotopy equivalent to a wedge of spheres, and give a combinatorial formula for the number of spheres in the wedge. This extends results of Berglund and Jöllenbeck on Golod rings and homotopy theoretical results of the first and third authors. We also establish a connection between minimally non-Golod rings and moment-angle complexes $\mathcal{Z}_{K}$ which are homotopy equivalent to a connected sum of sphere products. We go on to show that for any flag complex $K$ the loop spaces $\Omega \mathcal{Z}_{K}$ and $\Omega D J(K)$ are homotopy equivalent to a product of spheres and loops on spheres when localised rationally or at any prime $p \neq 2$.
\end{abstract}

\section{INTRODUCTION}

Moment-angle complexes are key players in the emerging field of toric topology, which lies on the borders between topology, algebraic and symplectic geometry, and combinatorics [8]. The moment-angle complex $\mathcal{Z}_{K}$, as a space with a torus action, appeared in work of Davis and Januszkiewicz [11] on topological generalisations of toric varieties. The homotopy orbit space of $\mathcal{Z}_{K}$ is the Davis-Januszkiewicz space $D J(K)$, which is a cellular model for the Stanley-Reisner ring $\mathbb{Z}[K]$, while the genuine orbit space of $\mathcal{Z}_{K}$ is the cone over the simplicial complex $K$. Buchstaber and the second author 7 introduced homotopy theoretical models of both the moment-angle complex $\mathcal{Z}_{K}$ and the Davis-Januszkiewicz space $D J(K)$ as a homotopy colimit construction of the product functor on the topological pairs $\left(D^{2}, S^{1}\right)$ and $\left(\mathbb{C} P^{\infty}, *\right)$ respectively, with the colimit taken over the face category of the \footnotetext{
2014 .

Received by the editors July 5, 2013 and, in revised form, July 7, 2014 and September 27,

2010 Mathematics Subject Classification. Primary 55P15; Secondary 13F55.

The first author's research was supported by the Leverhulme Trust (Research Project Grant RPG-2012-560).

The second author's research was supported by the Russian Foundation for Basic Research (grants 14-01-00537 and 14-01-92612-KO), Council for Grants of the President of the Russian Federation (grants NSh-4833.2014.1 and MD-111.2013.1), and by Dmitri Zimin's 'Dynasty' foundation.

The fourth author's research was supported in part by the Singapore Ministry of Education research grant (AcRF Tier 1 WBS No. R-146-000-190-112) and a grant (No. 11329101) of NSFC of China.
} 
simplicial complex $K$. Recently, homotopy theoretical generalisations of momentangle complexes and related spaces under the unifying umbrella of polyhedral products (see, for example, [1, 15], 16], 17]) have brought stable and unstable decomposition techniques to bear, and are leading to an improved understanding of toric spaces.

The homotopy theory of moment-angle complexes and polyhedral products in general has far reaching applications in combinatorial and homological algebra, in particular, in the study of face rings (or Stanley-Reisner rings) of simplicial complexes and more general monomial ideals.

In this paper we consider the following related homotopy theoretical and algebraic problems:

- identifying the homotopy type of the moment-angle complex $\mathcal{Z}_{K}$ for certain simplicial complexes $K$;

- describing the multiplication and higher Massey products in the Tor-algebra $H^{*}\left(\mathcal{Z}_{K}\right)=\operatorname{Tor}_{\mathbf{k}\left[v_{1}, \ldots, v_{m}\right]}(\mathbf{k}[K], \mathbf{k})$ of the face ring $\mathbf{k}[K]$;

- describing the Yoneda algebra $\operatorname{Ext}_{\mathbf{k}[K]}(\mathbf{k}, \mathbf{k})$ in terms of generators and relations;

- describing the structure of the Pontryagin algebra $H_{*}(\Omega D J(K))$ and its commutator subalgebra $H_{*}\left(\Omega \mathcal{Z}_{K}\right)$ via iterated and higher Whitehead (Samelson) products;

- identifying the homotopy type of the loop spaces $\Omega D J(K)$ and $\Omega \mathcal{Z}_{K}$.

The main objects and constructions are introduced in Section 2 together with some known preliminary results. In Section 3 we give topological interpretations of the Golod property of the face ring $\mathbf{k}[K]$. This ring is Golod if the multiplication in the Tor-algebra $H^{*}\left(\mathcal{Z}_{K}\right)=\operatorname{Tor}_{\mathbf{k}\left[v_{1}, \ldots, v_{m}\right]}(\mathbf{k}[K], \mathbf{k})$ is trivial, together with all higher Massey products (cf. [18, [19]). The topological interpretations are in terms of $H_{*}\left(\Omega \mathcal{Z}_{K}\right)$ being a free graded associative algebra, $H^{*}\left(\mathcal{Z}_{K}\right)$ having a trivial multiplication, and a certain identity holding for the Poincaré series of $H_{*}\left(\Omega \mathcal{Z}_{K}\right)$.

In Section 4 we concentrate on the case when $K$ is a flag complex. Our techniques allow for a complete solution of the problems above in the case of flag complexes. A flag complex $K$ is determined by its 1 -skeleton $K^{1}$. The Yoneda algebra $\operatorname{Ext}_{\mathbf{k}[K]}(\mathbf{k}, \mathbf{k}) \cong H_{*}(\Omega D J(K))$ has a simple presentation as a graph product algebra. In Theorem 4.3 we explicitly describe the minimal generating set of its commutator subalgebra $H_{*}\left(\Omega \mathcal{Z}_{K}\right)$ and the basis of the corresponding iterated commutators.

From the homotopy-theoretic point of view, particularly important momentangle complexes $\mathcal{Z}_{K}$ are those which have the homotopy type of a wedge of spheres. In this case the associative graded algebra $H_{*}\left(\Omega \mathcal{Z}_{K}\right)$ is free, and the multiplication in the Tor-algebra $H^{*}\left(\mathcal{Z}_{K}\right)=\operatorname{Tor}_{\mathbf{k}\left[v_{1}, \ldots, v_{m}\right]}(\mathbf{k}[K], \mathbf{k})$ is trivial, together with all higher Massey products, so the face ring $\mathbf{k}[K]$ is Golod. In Theorem 4.6 we show that for flag complexes $K$ the Golodness of $K$ is the precise algebraic criterion for $\mathcal{Z}_{K}$ being homotopy equivalent to a wedge of spheres. Using a result of Berglund and Jöllenbeck 4], this can be reformulated entirely in terms of the cup product: for a flag complex $K$, the moment-angle complex $\mathcal{Z}_{K}$ is homotopy equivalent to a wedge of spheres if and only if the cup product in $H^{*}\left(\mathcal{Z}_{K}\right)$ is trivial. Most importantly, there is a purely combinatorial description of the class of flag complexes $K$ for which $\mathcal{Z}_{K}$ is homotopy equivalent to a wedge of spheres: the 1-skeleton of 
such $K$ must be a chordal graph. This is an important concept in applied combinatorics and optimisation; the vertices in a chordal graph admit a perfect elimination ordering [14].

For general $K$, the Golod property of $\mathbf{k}[K]$ does not guarantee that $\mathcal{Z}_{K}$ is homotopy equivalent to a wedge of spheres. The reason is that for some Golod complexes $K$, the cohomology ring $H^{*}\left(\mathcal{Z}_{K} ; \mathbb{Z}\right)$ may contain non-trivial torsion (see Example 3.3. . Especially intriguing is that, for all known examples of Golod complexes $K$, the moment-angle complex $\mathcal{Z}_{K}$ is a co- $H$-space (in fact, a suspension) and this may as well be true in general (see Question 3.4).

The next homotopy type of $\mathcal{Z}_{K}$ which we consider is a connected sum of sphere products, where each summand is a product of exactly two spheres. Such a $\mathcal{Z}_{K}$ is obtained by attaching a top cell to a wedge of spheres along one commutator relation. The corresponding face ring $\mathbf{k}[K]$ is minimally non-Golod, and the commutator subalgebra $H_{*}\left(\Omega \mathcal{Z}_{K}\right)$ in the Yoneda algebra $\operatorname{Ext}_{\mathbf{k}[K]}(\mathbf{k}, \mathbf{k}) \cong H_{*}(\Omega D J(K))$ is a one-relator algebra. In the case of a flag simplicial complex $K$ the previous statement classifies minimally non-Golod Stanley-Reisner rings $\mathbf{k}[K]$, that is, $\mathbf{k}[K]$ is minimally non-Golod if and only if the moment-angle complex $\mathcal{Z}_{K}$ is homotopy equivalent to a connected sum of sphere products. It is an open question whether this classification criteria holds for a general simplicial complex (see Question 3.5).

In Section [5 we address the last problem in the list above. Our main result there is Theorem 5.3 which shows that for a flag $K$, both $\Omega \mathcal{Z}_{K}$ and $\Omega D J(K)$ are homotopy equivalent to products of spheres and loops of spheres when localised rationally or at any prime $p \neq 2$. We also show that the integral Pontryagin algebra $H_{*}\left(\Omega \mathcal{Z}_{K}\right)$ is torsion-free (Corollary [5.2).

In Section 6 we give a detailed illustration of many of the ideas and results of the paper in the case when $K$ is the boundary of a pentagon.

\section{Preliminaries}

Let $K$ be a finite simplicial complex on the set $[m]=\{1,2, \ldots, m\}$, that is, a collection of subsets $I=\left\{i_{1}, \ldots, i_{k}\right\} \subset[m]$ closed under inclusion. We refer to $I \in K$ as simplices or faces of $K$, and always assume that $\varnothing \in K$.

Assume we are given a set of $m$ topological pairs

$$
(\boldsymbol{X}, \boldsymbol{A})=\left\{\left(X_{1}, A_{1}\right), \ldots,\left(X_{m}, A_{m}\right)\right\}
$$

where $A_{i} \subset X_{i}$. For each simplex $I \in K$ we set

$$
(\boldsymbol{X}, \boldsymbol{A})^{I}=\left\{\left(x_{1}, \ldots, x_{m}\right) \in \prod_{i=1}^{m} X_{i} \mid x_{i} \in A_{i} \quad \text { for } i \notin I\right\} .
$$

The polyhedral product of $(\boldsymbol{X}, \boldsymbol{A})$ corresponding to $K$ is the following subset in $\prod_{i=1}^{m} X_{i}$ :

$$
(\boldsymbol{X}, \boldsymbol{A})^{K}=\bigcup_{I \in K}(\boldsymbol{X}, \boldsymbol{A})^{I}=\bigcup_{I \in K}\left(\prod_{i \in I} X_{i} \times \prod_{i \notin I} A_{i}\right) .
$$

In the case when all the pairs $\left(X_{i}, A_{i}\right)$ are the same, that is, $X_{i}=X$ and $A_{i}=A$ for $i=1, \ldots, m$, we use the notation $(X, A)^{K}$ for $(\boldsymbol{X}, \boldsymbol{A})^{K}$.

The main example of the polyhedral product is the moment-angle complex $\mathcal{Z}_{K}=$ $\left(D^{2}, S^{1}\right)^{K}\left[6\right.$, which is the key object of study in toric topology. The space $\mathcal{Z}_{K}$ has a natural coordinatewise action of the torus $T^{m}$, and it is a manifold whenever $K$ is a triangulation of a sphere. Other important cases of polyhedral products include 
$D J(K)=\left(\mathbb{C} P^{\infty}, *\right)^{K}$, which is referred to as the Stanley-Reisner space [6] or the Davis-Januszkiewicz space [22, and the complement of the complex coordinate subspace arrangement corresponding to $K$

$$
U(K)=\left(\mathbb{C}, \mathbb{C}^{*}\right)^{K}=\mathbb{C}^{m} \backslash \bigcup_{\left\{i_{1}, \ldots, i_{k}\right\} \notin K}\left\{z_{i_{1}}=\cdots=z_{i_{k}}=0\right\} .
$$

According to [6. Th. 5.2.5], there is a $T^{m}$-equivariant deformation retraction $U(K) \rightarrow \mathcal{Z}_{K}$. The spaces $\mathcal{Z}_{K}$ and $\left(\mathbb{C} P^{\infty}, *\right)^{K}$ are related by the following result.

Proposition 2.1 ([6, Cor. 3.4.5]). There is a homotopy fibration

$$
\mathcal{Z}_{K} \longrightarrow D J(K) \longrightarrow\left(\mathbb{C} P^{\infty}\right)^{m}
$$

that is, $\mathcal{Z}_{K}$ is the homotopy fibre of the canonical inclusion $D J(K) \rightarrow\left(\mathbb{C} P^{\infty}\right)^{m}$.

This fibration splits after looping

$$
\Omega D J(K) \simeq \Omega \mathcal{Z}_{K} \times T^{m}
$$

but this is not an $H$-space splitting. One can think of $\Omega \mathcal{Z}_{K}$ as the "commutator subgroup" of $\Omega D J(K)$, although this can be made precise only after passing to Pontryagin (loop homology) algebras.

Proposition 2.2 ([21, (8.2)]). There is an exact sequence of (non-commutative) algebras

$$
1 \longrightarrow H_{*}\left(\Omega \mathcal{Z}_{K} ; \mathbf{k}\right) \longrightarrow H_{*}(\Omega D J(K) ; \mathbf{k}) \longrightarrow \Lambda\left[u_{1}, \ldots, u_{m}\right] \longrightarrow 1
$$

where $\mathbf{k}$ is field or $\mathbb{Z}$, and $\Lambda\left[u_{1}, \ldots, u_{m}\right]$ is the exterior algebra on $m$ generators of degree one.

In what follows we shall often omit the coefficient ring $\mathbf{k}$ in the notation of (co)homology.

The exterior algebra $\Lambda\left[u_{1}, \ldots, u_{m}\right]$ can be thought of as the abelianisation of a largely non-commutative algebra $H_{*}(\Omega D J(K))$ (we expand on this below), so that $H_{*}\left(\Omega \mathcal{Z}_{K}\right)$ is its commutator subalgebra.

The face ring of $K$ (also known as the Stanley-Reisner ring) is defined as the quotient of the polynomial algebra $\mathbf{k}\left[v_{1}, \ldots, v_{m}\right]$ by the square-free monomial ideal generated by non-simplices of $K$

$$
\mathbf{k}[K]=\mathbf{k}\left[v_{1}, \ldots, v_{m}\right] /\left(v_{i_{1}} \cdots v_{i_{k}} \mid\left\{i_{1}, \ldots, i_{k}\right\} \notin K\right) .
$$

We make it graded by setting $\operatorname{deg} v_{i}=2$.

Theorem 2.3 ([11], 6, Prop. 3.4.3]). There is an isomorphism of graded commutative algebras

$$
H^{*}(D J(K) ; \mathbf{k}) \cong \mathbf{k}[K]
$$

for any coefficient ring $\mathbf{k}$.

The cohomology ring $H^{*}\left(\mathcal{Z}_{K} ; \mathbf{k}\right)$ and the Pontryagin algebra $H_{*}(\Omega D J(K) ; \mathbf{k})$ decode different homological invariants of the face ring $\mathbf{k}[K]$, as is stated next.

Theorem 2.4 ([6, Th. 5.3.4]). If $\mathbf{k}$ is a field, then there is an isomorphism of graded non-commutative algebras

$$
H_{*}(\Omega D J(K) ; \mathbf{k}) \cong \operatorname{Ext}_{\mathbf{k}[K]}(\mathbf{k}, \mathbf{k})
$$

where $\operatorname{Ext}_{\mathbf{k}[K]}(\mathbf{k}, \mathbf{k})$ is the Yoneda algebra of $\mathbf{k}[K]$. 
This is proved by applying the Adams cobar spectral sequence to the loop fibration $\Omega D J(K) \rightarrow \mathcal{P} D J(K) \rightarrow D J(K)$, where $\mathcal{P} D J(K)$ is the space of based paths in $D J(K)$ and using the formality of $D J(K)$.

Theorem 2.5 ([6], [2], [12]). If $\mathbf{k}$ is a field or $\mathbb{Z}$, then there are isomorphisms of (bi)graded commutative algebras

$$
\begin{aligned}
H^{*}\left(\mathcal{Z}_{K}\right) & \cong \operatorname{Tor}_{\mathbf{k}\left[v_{1}, \ldots, v_{m}\right]}(\mathbf{k}[K], \mathbf{k}) \\
& \cong H\left[\Lambda\left[u_{1}, \ldots, u_{m}\right] \otimes \mathbf{k}[K], d\right] \\
& \cong \bigoplus_{I \subset[m]} \widetilde{H}^{*}\left(K_{I}\right)
\end{aligned}
$$

Here, the second row is the cohomology of the differential bigraded algebra with bideg $u_{i}=(-1,2)$, bideg $v_{i}=(0,2)$ and $d u_{i}=v_{i}, d v_{i}=0$ (the Koszul complex). In the third row, $\widetilde{H}^{*}\left(K_{I}\right)$ denotes the reduced simplicial cohomology of the full subcomplex $K_{I} \subset K$ (the restriction of $K$ to $I \subset[m]$ ). The last isomorphism is the sum of isomorphisms

$$
H^{p}\left(\mathcal{Z}_{K}\right) \cong \sum_{I \subset[m]} \widetilde{H}^{p-|I|-1}\left(K_{I}\right)
$$

and the ring structure (the Hochster ring) is given by the maps

$$
H^{p-|I|-1}\left(K_{I}\right) \otimes H^{q-|J|-1}\left(K_{J}\right) \rightarrow H^{p+q-|I|-|J|-1}\left(K_{I \cup J}\right)
$$

which are induced by the canonical simplicial maps $K_{I \cup J} \rightarrow K_{I} * K_{J}$ for $I \cap J=\varnothing$ and zero otherwise.

In [16] several classes of complexes $K$ have been identified for which $\mathcal{Z}_{K}$ has homotopy type of a wedge of spheres. These include all skeleta of simplices, and the so-called shifted complexes. One special case which we shall refer to several times later is when $K$ is a disjoint union of finitely many vertices.

Theorem 2.6 ([15]). Let $K$ be the disjoint union of $m$ points. Then there is a homotopy equivalence

$$
\mathcal{Z}_{K} \simeq \bigvee_{\ell=2}^{m}\left(S^{\ell+1}\right)^{\vee(\ell-1)\left(\begin{array}{c}
m \\
\ell
\end{array}\right)} .
$$

Further, in [16] it was shown that there is a way to build new complexes $K$ whose corresponding $\mathcal{Z}_{K}$ is a wedge of spheres from existing ones.

Theorem 2.7 ([16, Th. 10.1]). Assume that $\mathcal{Z}_{K_{1}}$ and $\mathcal{Z}_{K_{2}}$ both have homotopy type of a wedge of spheres, and $K$ is obtained by attaching $K_{1}$ to $K_{2}$ along a common face. Then $\mathcal{Z}_{K}$ also has homotopy type of a wedge of spheres.

Corollary 2.8. Assume that there is an order $I_{1}, \ldots, I_{s}$ of the maximal faces of $K$ such that $\left(\bigcup_{j<k} I_{j}\right) \cap I_{k}$ is a single face for each $k=1, \ldots, s$. Then $\mathcal{Z}_{K}$ has homotopy type of a wedge of spheres.

\section{The Golod PROPERTY}

In this section we give topological interpretations of the Golod property. The face ring $\mathbf{k}[K]$ is called Golod (cf. [19]) if the multiplication and all higher Massey operations in $\operatorname{Tor}_{\mathbf{k}\left[v_{1}, \ldots, v_{m}\right]}(\mathbf{k}[K], \mathbf{k})$ are trivial. The Golod property can be defined for general graded or local Noetherian rings. Several combinatorial criteria for 
Golodness were given in 20]. We say that the simplicial complex $K$ is Golod if $\mathbf{k}[K]$ is a Golod ring. In view of Theorem 2.5, the Golod property is an algebraic approximation to the property of $\mathcal{Z}_{K}$ being homotopy equivalent to a wedge of spheres, although this approximation is not exact as Example 3.3 below shows. By a result of Berglund and Jöllenbeck [4, Th. 5.1], $K$ is a Golod complex if the multiplication in $\operatorname{Tor}_{\mathbf{k}\left[v_{1}, \ldots, v_{m}\right]}(\mathbf{k}[K], \mathbf{k})$ is trivial, i.e., there is no need to check the triviality of higher Massey products in the case of face rings.

Our main result in this section is Theorem 3.2 , but before stating this we give a more general result which is of independent interest. Recall that the Poincaré series of a graded k-module $A=\bigoplus_{i \geqslant 0} A^{i}$ is given by $P(A ; t)=\sum_{i \geqslant 0} \operatorname{dim} A^{i}$.

Proposition 3.1. Let $X$ be a simply-connected $C W$-complex such that $H_{*}(\Omega X ; \mathbf{k})$ is a graded free associative algebra, where $\mathbf{k}$ is a field. Then $H^{*}(X ; \mathbf{k})$ has trivial multiplication.

Proof. Let $Q=H_{>0}(\Omega X) /\left(H_{>0}(\Omega X) \cdot H_{>0}(\Omega X)\right)$ be the space of indecomposable elements, so that $H_{*}(\Omega X)=T\langle Q\rangle$ by assumption, where $T\langle Q\rangle$ denotes the free associative algebra on the graded k-module $Q$.

Consider the Rothenberg-Steenrod (bar) spectral sequence, which has $E_{2}$-term $E_{2}^{\mathrm{b}}=\operatorname{Tor}_{H_{*}(\Omega X)}(\mathbf{k}, \mathbf{k})$ and converges to $H_{*}(X)$. By assumption,

$$
E_{2}^{\mathrm{b}}=\operatorname{Tor}_{T\langle Q\rangle}(\mathbf{k}, \mathbf{k}) \cong \mathbf{k} \oplus Q
$$

as a $\mathbf{k}$-module. We therefore obtain the following inequalities for the Poincaré series:

$$
P\left(\Sigma^{-1} \widetilde{H}_{*}(X) ; t\right)=P\left(E_{\infty}^{\mathrm{b}} ; t\right)-1 \leqslant P\left(E_{2}^{\mathrm{b}} ; t\right)-1=P(Q ; t) .
$$

Now consider the Adams (cobar) spectral sequence, which has $E_{2}$-term $E_{2}^{\mathrm{c}}=$ Cotor $_{H_{*}(X)}(\mathbf{k}, \mathbf{k})$ and converges to $H_{*}(\Omega X)$. We have a series of inequalities:

$$
P\left(H_{*}(\Omega X) ; t\right)=P\left(E_{\infty}^{\mathrm{c}} ; t\right) \leqslant P\left(E_{2}^{\mathrm{c}} ; t\right) \leqslant P\left(T\left\langle\Sigma^{-1} \widetilde{H}_{*}(X)\right\rangle ; t\right) \leqslant P(T\langle Q\rangle ; t),
$$

where the second-to-last inequality follows from the cobar construction (it turns to equality when all differentials in the cobar construction on $H_{*}(X)$ are trivial), and the last inequality follows from (3.1). Now, $P\left(H_{*}(\Omega X) ; t\right)=P(T\langle Q\rangle ; t)$ by assumption, so all inequalities above turn into equalities, and both spectral sequences collapse at the $E_{2}$-term. It follows from the collapse of both spectral sequences that the homology map

$$
\widetilde{H}_{*}(\Sigma \Omega X)=\Sigma \widetilde{H}_{*}(\Omega X) \rightarrow \widetilde{H}_{*}(X)
$$

induced by the evaluation $\Sigma \Omega X \rightarrow X$ is onto. Consider the commutative diagram

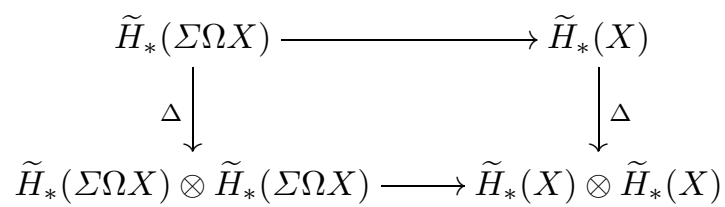

in which the vertical arrows are comultiplications, and the horizontal ones are surjective. Since $\Sigma \Omega X$ is a suspension, the left arrow is zero, hence, the right arrow is also zero. By duality, the multiplication in $H^{*}(X)$ is trivial. 
The Golod property of $K$ has the following topological interpretations.

Theorem 3.2. Let $\mathbf{k}$ be a field. The following conditions are equivalent:

(a) $H_{*}\left(\Omega \mathcal{Z}_{K}\right)$ is a graded free associative algebra;

(b) the multiplication in $H^{*}\left(\mathcal{Z}_{K}\right)$ is trivial;

(c) there is the following identity for the Poincaré series:

$$
P\left(H_{*}\left(\Omega \mathcal{Z}_{K}\right) ; t\right)=\frac{1}{1-P\left(\Sigma^{-1} \widetilde{H}^{*}\left(\mathcal{Z}_{K}\right) ; t\right)},
$$

where $\Sigma^{-1}$ denotes the desuspension of a graded $\mathbf{k}$-module.

Proof. The implication (a) $\Rightarrow$ (b) holds by Proposition 3.1

To prove the implication $(\mathrm{b}) \Rightarrow(\mathrm{c})$ we use the above mentioned result [4, Th. 5.1], according to which if the product in $H^{*}\left(\mathcal{Z}_{K}\right)=\operatorname{Tor}_{\mathbf{k}\left[v_{1}, \ldots, v_{m}\right]}(\mathbf{k}[K], \mathbf{k})$ is trivial, then all higher Massey operations are also trivial, that is, $\mathbf{k}[K]$ is Golod. By the alternative definition of the Golod property [19, $\mathbf{k}[K]$ is Golod if and only if the following identity for the Poincaré series holds:

$$
P\left(\operatorname{Ext}_{\mathbf{k}[K]}(\mathbf{k}, \mathbf{k}) ; t\right)=\frac{(1+t)^{m}}{1-\sum_{i, j>0} \operatorname{dim} \operatorname{Tor}_{\mathbf{k}\left[v_{1}, \ldots, v_{m}\right]}^{-i, 2 j}(\mathbf{k}[K], \mathbf{k}) t^{-i+2 j-1}} .
$$

Using Theorems 2.4 and 2.5, we rewrite this as

$$
P\left(H_{*}(\Omega D J(K)) ; t\right)=\frac{P\left(H_{*}\left(T^{m}\right) ; t\right)}{1-P\left(\Sigma^{-1} \widetilde{H}^{*}\left(\mathcal{Z}_{K}\right) ; t\right)} .
$$

Since $\Omega D J(K) \simeq \Omega \mathcal{Z}_{K} \times T^{m}$, the above identity is equivalent to that of (c).

To prove the implication (c) $\Rightarrow$ (a) we observe that

$$
\frac{1}{1-P\left(\Sigma^{-1} \widetilde{H}^{*}\left(\mathcal{Z}_{K}\right) ; t\right)}=P\left(T\left\langle\Sigma^{-1} \widetilde{H}_{*}\left(\mathcal{Z}_{K}\right)\right\rangle ; t\right)
$$

so the identity from (c) is equivalent to $P\left(H_{*}\left(\Omega \mathcal{Z}_{K}\right) ; t\right)=P\left(T\left\langle\Sigma^{-1} \widetilde{H}_{*}\left(\mathcal{Z}_{K}\right)\right\rangle\right)$. Hence, all differentials in the cobar construction on $H_{*}\left(\mathcal{Z}_{K}\right)$ are trivial, which implies that $H_{*}\left(\Omega \mathcal{Z}_{K}\right)$ is a free associative algebra on $\Sigma^{-1} \widetilde{H}_{*}\left(\mathcal{Z}_{K}\right)$.

The conditions of Theorem 3.2 do not guarantee that $\mathcal{Z}_{K}$ is homotopy equivalent to a wedge of spheres. One reason is that $H^{*}\left(\mathcal{Z}_{K} ; \mathbb{Z}\right)$ may contain arbitrary torsion. This follows easily from Theorem 2.5. since $\widetilde{H}^{*}(K)$ is a direct summand in $H^{*}\left(\mathcal{Z}_{K}\right)$, one may take $K$ to be a triangulation of a space with torsion in cohomology. The simplest example is the 6 -vertex triangulation of $\mathbb{R} P^{2}$.

Example 3.3. Let $K$ be the simplicial complex shown in Figure 1, where the vertices with the same labels are identified, and the boundary edges are identified according to the orientation shown. A calculation using Theorem 2.5 shows that the non-trivial cohomology groups of $\mathcal{Z}_{K}$ are given by

$$
H^{0}=\mathbb{Z}, \quad H^{5}=\mathbb{Z}^{10}, \quad H^{6}=\mathbb{Z}^{15}, \quad H^{7}=\mathbb{Z}^{6}, \quad H^{9}=\mathbb{Z} / 2 .
$$

Therefore, all products and Massey products vanish for dimensional reasons, so $K$ is Golod (over any field). Nevertheless, $\mathcal{Z}_{K}$ is not homotopy equivalent to a wedge of spheres because of the torsion. In particular, in this example we have

$$
\mathcal{Z}_{K} \simeq\left(S^{5}\right)^{\vee 10} \vee\left(S^{6}\right)^{\vee 15} \vee\left(S^{7}\right)^{\vee 6} \vee \Sigma^{7} \mathbb{R} P^{2}
$$




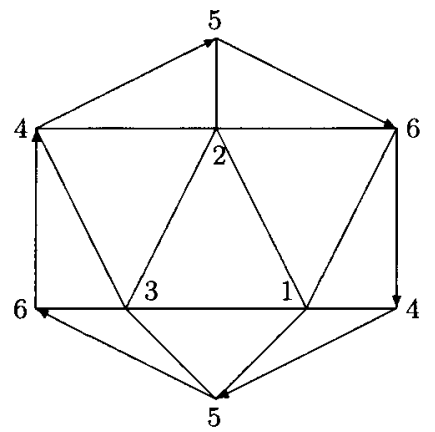

Figure 1. 6-vertex triangulation of $\mathbb{R} P^{2}$.

where $X^{\vee k}$ denotes the $k$-fold wedge of $X$. For, if we regard $\mathcal{Z}_{K}$ as a $C W$-complex built up by attaching $k$-cells to the $(k-1)$-skeleton for $6 \leqslant k \leqslant 9$, then the attaching maps are all in the stable range. But stably these attaching maps are all null homotopic since, by [1, the homotopy equivalence in (3.2) holds after one suspension. Therefore the attaching maps are null homotopic, and so (3.2) holds without having to suspend.

Question 3.4. Assume that $H^{*}\left(\mathcal{Z}_{K}\right)$ has trivial multiplication, so that $K$ is Golod, over any field. Is it true that $\mathcal{Z}_{K}$ is a co- $H$-space, or even a suspension, as in all known examples?

Denote by $K_{\widehat{i}}$ the restriction of $K$ to the set of vertices $[m] \backslash\{i\}$, that is, $K_{\widehat{i}}=$ $\{J \in K \mid i \notin J\}$. It follows from the description of the product in $H^{*}\left(\mathcal{Z}_{K}\right)$ in Theorem 2.5 that if $K$ is Golod, then $K_{\widehat{i}}$ is also Golod. Following [4, we refer to $K$ as a minimally non-Golod complex if $K$ is not Golod, but $K_{\widehat{i}}$ is Golod for each $i$.

The condition for $K$ to be minimally non-Golod is an "algebraic approximation" of the topological condition for $\mathcal{Z}_{K}$ to be homeomorphic to a connected sum of sphere products, with two spheres in each product. In what follows, whenever we say that $\mathcal{Z}_{K}$ is a connected sum of sphere products, we mean that each summand is a product of exactly two spheres. (In fact, there is no known example of $\mathcal{Z}_{K}$ which is homeomorphic to a non-trivial connected sum of sphere products with more than two spheres in at least one product.)

To justify the term "algebraic approximation", the following question needs to be positively answered.

Question 3.5. Is it true that if $\mathcal{Z}_{K}$ is a connected sum of sphere products, then $K$ is minimally non-Golod?

Examples of minimally non-Golod complexes include the boundary complexes of polygons and, more generally, stacked polytopes different from simplices 4, Th. 6.19]. For all these cases it is known that $\mathcal{Z}_{K}$ is homeomorphic to a connected sum of sphere products, due to a result of McGavran (cf. [5, Th. 6.3]; see also Section 6 below).

\section{The CASE OF A FLAG COMPLEX}

A missing face (or a minimal non-face) of $K$ is a subset $I \subset[m]$ such that $I \notin K$, but every proper subset of $I$ is a simplex of $K$. A simplicial complex $K$ is called a 
flag complex if each of its missing faces has two vertices. Equivalently, $K$ is flag if any set of vertices of $K$ which are pairwise connected by edges spans a simplex.

In the case of flag complexes $K$ we shall show that the "algebraic approximations" from the previous section are precise criterions for the appropriate topological properties: $\mathcal{Z}_{K}$ is a wedge of spheres precisely when $K$ is Golod, and $\mathcal{Z}_{K}$ is a connected sum of sphere products if and only if $K$ is minimally non-Golod.

There is the following description of $H_{*}(\Omega D J(K))=\operatorname{Ext}_{\mathbf{k}[K]}(\mathbf{k}, \mathbf{k})$ for flag $K$.

Theorem 4.1 ([21, Th. 9.3]). For any flag complex $K$, there is an isomorphism

$$
H_{*}(\Omega D J(K) ; \mathbf{k}) \cong T\left\langle u_{1}, \ldots, u_{m}\right\rangle /\left(u_{i}^{2}=0, u_{i} u_{j}+u_{j} u_{i}=0 \text { for }\{i, j\} \in K\right)
$$

where $\mathbf{k}$ is a field and $T\left\langle u_{1}, \ldots, u_{m}\right\rangle$ is the free associative algebra on $m$ generators of degree 1 .

Remark. The theorem above is formulated in 21 with $\mathbb{Q}$-coefficients, but the argument (using the Adams cobar construction and a result of Fröberg [13] on quadratic duality) works for arbitrary field.

Algebra (4.1) may be viewed as a colimit (in the category of non-commutative associative algebras) of a diagram of algebras over the face category of $K$, which assigns to each face $I \in K$ the exterior algebra $\Lambda\left[u_{i}: i \in I\right]$. Another way to see this algebra is to assign a generator $u_{i}$ satisfying $u_{i}^{2}=0$ to each vertex of $K$, and think of each edge of $K$ as a commutativity relation between the corresponding $u_{i}$ 's. The resulting algebra is determined by the 1-skeleton (graph) of $K$, which is not surprising since $K$ is flag. In the non-flag case higher brackets appear, corresponding to higher Samelson products in $\Omega D J(K)$, and the colimit above has to be replaced by a homotopy colimit; see [21, $\S 8$ for the details.

Algebra (4.1) is also known as the graph product algebra corresponding to the 1-skeleton of $K$. Its group-theoretic analogues are right-angled Artin and Coxeter groups; in fact the polyhedral products of the form $\left(\mathbb{R} P^{\infty}, *\right)^{K}$ and $\left(S^{1}, *\right)^{K}$ respectively are the classifying spaces of these groups in the flag case (cf. [22, §4]).

The $f$-vector of $K$ is given by $\boldsymbol{f}(K)=\left(f_{0}, \ldots, f_{n-1}\right)$ where $f_{i}$ is the number of $i$-dimensional faces and $n-1=\operatorname{dim} K$. The $h$-vector $\boldsymbol{h}(K)=\left(h_{0}, h_{1}, \ldots, h_{n}\right)$ is defined from the relation

$$
h_{0} t^{n}+h_{1} t^{n-1}+\cdots+h_{n}=(t-1)^{n}+f_{0}(t-1)^{n-1}+\cdots+f_{n-1} .
$$

The $h$-vector is symmetric for sphere triangulations $K$; the equations $h_{i}=h_{n-i}$ are known as the Dehn-Sommerville relations.

As another application of quadratic duality, the Poincaré series of $H_{*}\left(\Omega \mathcal{Z}_{K}\right)$ can be calculated explicitly in terms of the face numbers of $K$ in the flag case.

Proposition 4.2 ([21, Prop. 9.5]). For any flag complex $K$, we have

$$
P\left(H_{*}\left(\Omega \mathcal{Z}_{K}\right) ; t\right)=\frac{1}{(1+t)^{m-n}\left(1-h_{1} t+\cdots+(-1)^{n} h_{n} t^{n}\right)} .
$$

We now go further by identifying a minimal set of multiplicative generators in $H_{*}\left(\Omega \mathcal{Z}_{K}\right)$ as a specific set of iterated commutators of the $u_{i}$.

Theorem 4.3. Assume that $K$ is flag and $\mathbf{k}$ is a field. The algebra $H_{*}\left(\Omega \mathcal{Z}_{K} ; \mathbf{k}\right)$, viewed as the commutator subalgebra (4.1) via exact sequence (2.1), is multiplicatively generated by $\sum_{I \subset[m]} \operatorname{dim} \widetilde{H}^{0}\left(K_{I}\right)$ iterated commutators of the form

$$
\left[u_{j}, u_{i}\right], \quad\left[u_{k_{1}},\left[u_{j}, u_{i}\right]\right], \quad \cdots, \quad\left[u_{k_{1}},\left[u_{k_{2}}, \cdots\left[u_{k_{m-2}},\left[u_{j}, u_{i}\right]\right] \cdots\right]\right]
$$


where $k_{1}<k_{2}<\cdots<k_{p}<j>i, k_{s} \neq i$ for any $s$, and $i$ is the smallest vertex in a connected component not containing $j$ of the subcomplex $K_{\left\{k_{1}, \ldots, k_{p}, j, i\right\}}$. Furthermore, this multiplicative generating set is minimal, that is, the commutators above form a basis in the submodule of indecomposables in $H_{*}\left(\Omega \mathcal{Z}_{K}\right)$.

Remark. To help clarify the statement of Theorem 4.3, it is useful to consider which brackets $\left[u_{j}, u_{i}\right]$ are in the list of multiplicative generators for $H_{*}\left(\Omega \mathcal{Z}_{K} ; \mathbf{k}\right)$. If $\{j, i\} \in K$, then $i$ and $j$ are in the same connected component of the subcomplex $K_{\{j, i\}}$, so $\left[u_{j}, u_{i}\right]$ is not a multiplicative generator. On the other hand, if $\{j, i\} \notin K$, then the subcomplex $K_{\{j, i\}}$ consists of the two distinct points $i$ and $j$, and $i$ is the smallest vertex in its connected component of $K_{\{j, i\}}$ which does not contain $j$, so $\left[u_{j}, u_{i}\right]$ is a multiplicative generator. In Section [6 the example where $K$ is a pentagon is worked out in detail, and in particular, a complete list of multiplicative generators for $H_{*}\left(\Omega \mathcal{Z}_{K} ; \mathbf{k}\right)$ is given.

Proof. We observe that, for a given $I=\left\{k_{1}, \ldots, k_{p}, j, i\right\}$, the number of the commutators containing all $u_{k_{1}}, \ldots, u_{k_{p}}, u_{j}, u_{i}$ in the set above is equal to $\operatorname{dim} \widetilde{H}^{0}\left(K_{I}\right)$ (one less than the number of connected components in $K_{I}$ ), so there are indeed $\sum_{I \subset[m]} \operatorname{dim} \widetilde{H}^{0}\left(K_{I}\right)$ commutators in total.

We first prove a particular case of the statement, corresponding to $K$ consisting of $m$ disjoint points. This result may be of independent algebraic interest, as it is an analogue of the description of a basis in the commutator subalgebra of a free algebra, given by Cohen and Neisendorfer [10].

Lemma 4.4. Let $A$ be the commutator subalgebra of $T\left\langle u_{1}, \ldots, u_{m}\right\rangle /\left(u_{i}^{2}=0\right)$, that is, the algebra defined by the exact sequence

$$
1 \longrightarrow A \longrightarrow T\left\langle u_{1}, \ldots, u_{m}\right\rangle /\left(u_{i}^{2}=0\right) \longrightarrow \Lambda\left[u_{1}, \ldots, u_{m}\right] \longrightarrow 1
$$

where $\operatorname{deg} u_{i}=1$. Then $A$ is a free associative algebra minimally generated by the iterated commutators of the form

$$
\left[u_{j}, u_{i}\right], \quad\left[u_{k_{1}},\left[u_{j}, u_{i}\right]\right], \quad \cdots, \quad\left[u_{k_{1}},\left[u_{k_{2}}, \cdots\left[u_{k_{m-2}},\left[u_{j}, u_{i}\right]\right] \cdots\right]\right]
$$

where $k_{1}<k_{2}<\cdots<k_{p}<j>i$ and $k_{s} \neq i$ for any $s$. Here, the number of commutators of length $\ell$ is equal to $(\ell-1)\left(\begin{array}{c}m \\ \ell\end{array}\right)$.

Proof. Let $S$ be the set of commutators in the statement of the lemma. Let $B$ denote the commutator algebra of a free algebra on $m$ generators, that is, the algebra kernel of the map $T\left\langle u_{1}, \ldots, u_{m}\right\rangle \rightarrow \Lambda\left[u_{1}, \ldots, u_{m}\right]$. By [10], $B$ is a free algebra generated by the commutators of the same form $\left[u_{k_{1}},\left[u_{k_{2}}, \cdots\left[u_{k_{p}},\left[u_{j}, u_{i}\right]\right] \cdots\right]\right]$, but with the conditions $k_{1}<k_{2}<\cdots<k_{p}<j \geqslant i$ only. We therefore get a larger set $T$ of commutators, in which $u_{k}$ may repeat. However, note that the inequalities on the indices imply that if $u_{k}$ repeats within a specified commutator, it does so only once. We have $S \subseteq T$ and wish to show that any commutator in $T-S$ is excluded from the multiplicative generating set of the quotient $T\left\langle u_{1}, \ldots, u_{m}\right\rangle /\left(u_{i}^{2}=0\right)$. To see this, induct on the length of the commutators, beginning with $\left[u_{k}, u_{k}\right]=2 u_{k}^{2}=0$. Suppose the commutators of length $<n$ in $T$ have had any commutator with a repeating $u_{k}$ excluded from the generating set of $T\left\langle u_{1}, \ldots, u_{m}\right\rangle /\left(u_{i}^{2}=0\right)$. Choose a commutator of length $n$ with some $u_{k}$ repeating. Observe that it suffices to consider commutators of the form $\left[u_{k},\left[u_{k_{2}}, \cdots\left[u_{k_{p}},\left[u_{j}, u_{k}\right]\right] \cdots\right]\right]$, which we write as $\left[u_{k},\left[u_{k_{2}}, c\right]\right]$ for $c=\left[u_{k_{3}}, \cdots\left[u_{k_{p}},\left[u_{j}, u_{k}\right]\right] \cdots\right]$. By the Jacobi identity, $\left[u_{k},\left[u_{k_{2}}, c\right]\right]=$ $\pm\left[c,\left[u_{k}, u_{k_{2}}\right]\right] \pm\left[u_{k_{2}},\left[c, u_{k}\right]\right]$. Rewriting to conform to the restrictions on the indices 
in the basis for $B$, we obtain $\left[u_{k},\left[u_{k_{2}}, c\right]\right]= \pm\left[c,\left[u_{k_{2}}, u_{k}\right]\right] \pm\left[u_{k_{2}},\left[u_{k}, c\right]\right]$. The first term on the right is a commutator of two elements of lower length in $S$. The second term on the right has $\left[u_{k}, c\right]$ excluded from the multiplicative generating set of $T\left\langle u_{1}, \ldots, u_{m}\right\rangle /\left(u_{i}^{2}=0\right)$ by inductive hypothesis, since $u_{k}$ appears in $c$. Therefore $\left[u_{k},\left[u_{k_{2}}, c\right]\right]$ is not a multiplicative generator of $T\left\langle u_{1}, \ldots, u_{m}\right\rangle /\left(u_{i}^{2}=0\right)$.

Now observe that the set of commutators $S$ generates $A$ multiplicatively, since $A$ is a quotient of $B /\left(u_{i}^{2}=0\right)$. To show that $A$ is a free algebra, and the given generator set is minimal, we use a topological argument. We have that $A=H_{*}\left(\Omega \mathcal{Z}_{K}\right)$ where $K$ is a disjoint union of $m$ points. By Theorem 2.6, $\mathcal{Z}_{K}$ is homotopy equivalent to the wedge of spheres $\bigvee_{\ell=2}^{m}\left(S^{\ell+1}\right)^{\vee(\ell-1)}\left(\begin{array}{c}m \\ \ell\end{array}\right)$. The Bott-Samelson Theorem implies that $A=H_{*}\left(\Omega \mathcal{Z}_{K}\right)$ is a free algebra, and the number of generators in each degree $\ell$ agrees with the number of given commutators of length $\ell$.

To complete the proof of Theorem 4.3 we must deal with how the remaining relations in (4.1), those of the form $u_{i} u_{j}+u_{j} u_{i}=0$ if $\{i, j\} \in K$, affect the iterated commutators listed in Lemma 4.4. Note that $u_{i} u_{j}+u_{j} u_{i}=\left[u_{i}, u_{j}\right]$ and that no $u_{k}$ repeats in any of the iterated commutators listed in Lemma 4.4.

Assume that $i, i^{\prime}$ are vertices in the same connected component of $K$. Then there are vertices $i_{1}=i, i_{2}, \ldots, i_{k-1}, i_{k}=i^{\prime}$ for some $k$ with the property that the edges $\left\{i_{1}, i_{2}\right\}, \ldots,\left\{i_{k-1}, i_{k}\right\}$ are all in $K$. Arguing inductively as in the proof of Lemma 4.4, the Jacobi identity implies that any iterated commutator of length $l$ involving all $u_{i_{1}}, \ldots, u_{i_{k}}$ can be rewritten as a sum of iterated commutators formed from iterated commutators of lengths $<l$. In particular, if $K$ is connected (with $m$ vertices), then any iterated commutator of length $m$ is zero modulo commutators of lesser length.

Continuing, suppose that we are given an index set $I=\left\{k_{1}, \ldots, k_{p}, j, i\right\}$ with $k_{1}<k_{2}<\cdots<k_{p}<j>i$ and $k_{s} \neq i$ for any $s$. Consider iterated commutators of length $p+2$ involving one occurrence of $u_{k}$ for each $k \in I$. One example is $\left[u_{k_{1}},\left[u_{k_{2}}, \cdots\left[u_{k_{p}},\left[u_{j}, u_{i}\right]\right] \cdots\right]\right]$. Observe that the restrictions on the order of the indices imply that the only other examples occur by interchanging $u_{i}$ and $u_{k_{l}}$ provided $k_{l-1}<i<k_{l+1}$. Now if $i, j$ are in the same connected component of $K_{I}$, then $\left[u_{k_{1}},\left[u_{k_{2}}, \cdots\left[u_{k_{p}},\left[u_{j}, u_{i}\right]\right] \cdots\right]\right]=0$ modulo iterated commutators of lesser length, by the argument in the previous paragraph applied to $K_{I}$. So to obtain non-trivial commutators we require that $i, j$ appear in different components. Also, if $\left\{k_{l_{1}}, \ldots, k_{l_{r}}\right\}$ is the subset of $\left\{k_{1}, \ldots, k_{p}\right\}$ which lie in the same connected component of $K_{I}$ as $i$, then the iterated commutators $\left[u_{k_{1}},\left[u_{k_{2}}, \cdots, u_{k_{l_{t}-1}},\left[u_{i},\left[u_{k_{l_{t}+1}}, \cdots\left[u_{k_{p}},\left[u_{j}, u_{k_{l_{t}}}\right]\right] \cdots\right]\right]\right.\right.$ and $\left[u_{k_{1}},\left[u_{k_{2}}, \cdots\left[u_{k_{p}}\right.\right.\right.$, $\left.\left.\left.\left[u_{j}, u_{i}\right]\right] \cdots\right]\right]$ can be identified modulo iterated commutators of lesser lengths. So to enumerate the one independent iterated commutator, we use the convention of writing $\left[u_{k_{1}},\left[u_{k_{2}}, \cdots\left[u_{k_{p}},\left[u_{j}, u_{i}\right]\right] \cdots\right]\right]$ where $i$ is the smallest vertex in its connected component within $K_{I}$. This leaves us with precisely the set of iterated commutators in the statement of the theorem.

At this point, we have shown that the set of iterated commutators in the statement of the theorem multiplicatively generates $H_{*}\left(\Omega \mathcal{Z}_{K}\right)$. It remains to show that this is a minimal generating set. To see this, it suffices to show that if $I=$ $\left\{k_{1}, \ldots, k_{p}, j, i\right\}$ where $k_{1}<\cdots<k_{p}<j>i$, then the remaining iterated commutators on this index set are algebraically independent. Let $\left\{k_{l_{1}}, \ldots, k_{l_{r}}\right\}$ be the subset of $\left\{k_{1}, \ldots, k_{p}\right\}$ whose elements lie in the same connected component of $K_{I}$ as $i$. Let 
$K_{\widehat{I}}$ be the full subcomplex of $K_{I}$ on the vertex set $I-\left\{k_{l_{1}}, \ldots, k_{l_{r}}\right\}$. There is a projection $K_{I} \rightarrow K_{\widehat{I}}$. Observe that the connected component of $K_{\widehat{I}}$ containing the vertex $i$ is precisely the singleton $\{i\}$, and there is a one-to-one correspondence between the remaining iterated commutators of the form $\left[u_{k_{1}},\left[u_{k_{2}}, \cdots\left[u_{k_{p}},\left[u_{j}, u_{i}\right]\right] \cdots\right]\right]$ in $H_{*}\left(\Omega D J\left(K_{I}\right)\right)$ and the iterated commutators of length $(p+2)-r$ in $H_{*}\left(\Omega D J\left(K_{\widehat{I}}\right)\right)$ formed by deleting the elements $u_{k_{l}}$ whenever $k_{l} \in\left\{k_{l_{i}}, \ldots, k_{l_{r}}\right\}$. The latter set is algebraically independent since, topologically, $D J\left(K_{\widehat{I}}\right)$ is the wedge $\mathbb{C} P^{\infty} \vee$ $D J\left(K_{\widehat{I}}-\{i\}\right)$, and the iterated commutators correspond to independent Whitehead products in $\Sigma \Omega \mathbb{C} P^{\infty} \wedge \Omega D \simeq \Sigma S^{1} \wedge \Omega D$, where $D=D J\left(K_{\widehat{I}}-\{i\}\right)$. Hence the former set is algebraically independent, as required.

We now come to identifying the class of flag complexes $K$ for which $\mathcal{Z}_{K}$ has homotopy type of a wedge of spheres.

Let $\Gamma$ be a graph on the vertex set $[m]$. A clique of $\Gamma$ is a subset $I$ of vertices such that every two vertices in $I$ are connected by an edge. Obviously, each flag complex $K$ is the clique complex of its 1 -skeleton $\Gamma=K^{1}$, that is, the simplicial complex formed by filling in each clique of $\Gamma$ by a face.

A graph $\Gamma$ is called chordal if each of its cycles with $\geqslant 4$ vertices has a chord (an edge joining two vertices that are not adjacent in the cycle). Equivalently, a chordal graph is a graph with no induced cycles of length more than three.

The following result gives an alternative characterisation of chordal graphs.

Theorem 4.5 ([14]). A graph is chordal if and only if its vertices can be ordered in such a way that, for each vertex $i$, the lesser neighbours of $i$ form a clique.

Such an order of vertices is called a perfect elimination ordering.

Theorem 4.6. Let $K$ be a flag complex and $\mathbf{k}$ a field. The following conditions are equivalent:

(a) $\mathbf{k}[K]$ is a Golod ring;

(b) the multiplication in $H^{*}\left(\mathcal{Z}_{K}\right)$ is trivial;

(c) $\Gamma=K^{1}$ is a chordal graph;

(d) $\mathcal{Z}_{K}$ has homotopy type of a wedge of spheres.

Proof. (a) $\Rightarrow$ (b) This is by definition of the Golod property and Theorem 2.5.

(b) $\Rightarrow$ (c) Assume that $K^{1}$ is not chordal, and choose an induced chordless cycle $I$ with $|I| \geqslant 4$. Then the full subcomplex $K_{I}$ is the same cycle (the boundary of an $|I|$ gon), and therefore $\mathcal{Z}_{K_{I}}$ is a connected sum of sphere products. Hence, $H^{*}\left(\mathcal{Z}_{K_{I}}\right)$ has non-trivial products (this can also be seen directly by using Theorem 2.5). Then, by Theorem 2.5, the same non-trivial products appear in $H^{*}\left(\mathcal{Z}_{K}\right)$.

$(\mathrm{c}) \Rightarrow(\mathrm{d})$ Assume that the vertices of $K$ are in perfect elimination order. We assign to each vertex $i$ the clique $I_{i}$ consisting of $i$ and the lesser neighbours of $i$. Each maximal face of $K$ (that is, each maximal clique of $K^{1}$ ) is obtained in this way, so we get an induced order on the maximal faces: $I_{i_{1}}, \ldots, I_{i_{s}}$. Then, for each $k=$ $1, \ldots, s$, the simplicial complex $\bigcup_{j<k} I_{i_{j}}$ is flag (since it is the full subcomplex $K_{\left\{1,2, \ldots, i_{k-1}\right\}}$ in a flag complex). The intersection $\left(\bigcup_{j<k} I_{i_{j}}\right) \cap I_{i_{k}}$ is a clique, so it is a face of $\bigcup_{j<k} I_{i_{j}}$. Therefore, $\mathcal{Z}_{K}$ has homotopy type of a wedge of spheres by Corollary 2.8

$(d) \Rightarrow$ (a) This is by definition of the Golod property and the fact that the cohomology of the wedge of spheres contains only trivial cup and Massey products. 
Remark. The equivalence of (a), (b) and (c) was proved in [4, Th. 6.5].

All the implications in the above proof except $(\mathrm{c}) \Rightarrow(\mathrm{d})$ are valid for arbitrary $K$, with the same arguments. However, $(\mathrm{c}) \Rightarrow(\mathrm{d})$ fails in the non-flag case; Example 3.3 is a counterexample.

Corollary 4.7. Assume that $K$ is flag with $m$ vertices and $\mathcal{Z}_{K}$ has homotopy type of a wedge of spheres. Then

(a) the maximal dimension of spheres in the wedge is $m+1$;

(b) the number of spheres of dimension $\ell+1$ in the wedge is given by $\sum_{|I|=\ell} \operatorname{dim} \widetilde{H}^{0}\left(K_{I}\right)$, for $2 \leqslant \ell \leqslant m ;$

(c) $H^{i}\left(K_{I}\right)=0$ for $i>0$ and all $I$.

Proof. If $\mathcal{Z}_{K}$ is a wedge of spheres, then $H_{*}\left(\Omega \mathcal{Z}_{K}\right)$ is a free algebra on generators described by Theorem 4.3, which implies (a) and (b). It also follows that $H^{*}\left(\mathcal{Z}_{K}\right) \cong$ $\bigoplus_{I \subset[m]} \widetilde{H}^{0}\left(K_{I}\right)$. On the other hand, $H^{*}\left(\mathcal{Z}_{K}\right) \cong \bigoplus_{I \subset[m]} \widetilde{H}^{*}\left(K_{I}\right)$ by Theorem 2.5. whence $(\mathrm{c})$ follows.

Theorem 4.8. Assume that $K$ is flag and $\mathbf{k}$ a field. The following conditions are equivalent:

(a) $K$ is minimally non-Golod;

(b) $\mathcal{Z}_{K}$ is homeomorphic to a connected sum of sphere products.

Proof. Indeed, if $K$ is flag and minimally non-Golod, then it is the boundary of an $m$-gon with $m \geqslant 4$.

\section{The homotopy type of $\Omega_{\mathcal{Z}_{K}}$ When $K$ IS FLAG}

In general, the homotopy type of $\mathcal{Z}_{K}$ when $K$ is a flag complex may not be easy to determine. We have shown that $\mathcal{Z}_{K}$ has the homotopy type of a wedge of spheres if $K$ is Golod, and $\mathcal{Z}_{K}$ has the homotopy type of a connected sum of sphere products if $K$ is minimally non-Golod. Beyond these two classes, it is not clear what the homotopy type of $\mathcal{Z}_{K}$ may be. However, we will show in Theorem 5.3 that the homotopy type of $\Omega \mathcal{Z}_{K}$ localised away from 2 is a product of spheres and loops on spheres.

To begin, suppose that $K$ is a flag complex on $m$ vertices. Let $\bar{K}$ be the disjoint union of the $m$ vertices. Then the inclusion

$$
i: \bar{K} \longrightarrow K
$$

induces an inclusion

$$
D J(i): D J(\bar{K})=\bigvee_{j=1}^{m} \mathbb{C} P^{\infty} \longrightarrow D J(K)
$$

and we obtain a homotopy pullback diagram

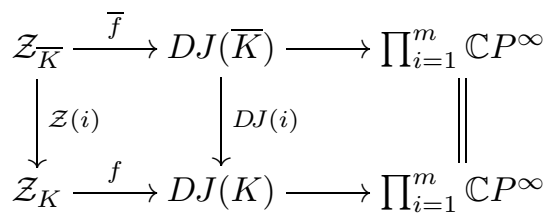

which defines the maps $\mathcal{Z}(i), \bar{f}$ and $f$.

It is useful to have some initial algebraic information. 
Lemma 5.1. Let $f: X \longrightarrow Y$ be a map between two simply-connected spaces. If $H_{*}(\Omega X ; \mathbb{Z})$ is torsion-free and $(\Omega f)_{*}$ is onto for coefficients in any field, then $H_{*}(\Omega Y ; \mathbb{Z})$ is also torsion-free.

Proof. Suppose $H_{*}(\Omega Y ; \mathbb{Z})$ is not torsion-free. Then there is a prime $p$ and elements $b, \bar{b} \in H_{*}(\Omega Y ; \mathbb{Z} / p \mathbb{Z})$ such that $\beta^{r} \bar{b}=b$, where $\beta^{r}$ is the $r^{t h}$-Bockstein. As $(\Omega f)_{*}$ is onto in mod- $p$ homology, there are elements $a, \bar{a} \in H_{*}(\Omega X ; \mathbb{Z} / p \mathbb{Z})$ such that $(\Omega f)_{*}(a)=b$ and $(\Omega f)_{*}(\bar{a})=\bar{b}$. As $\beta^{r}$ commutes with $(\Omega f)_{*}$, we obtain

$$
(\Omega f)_{*}\left(\beta^{r} \bar{a}\right)=\beta^{r}(\Omega f)_{*}(\bar{a})=\beta^{r} \bar{b}=b,
$$

implying that $\beta^{r} \bar{a} \neq 0$. This contradicts the fact that $H_{*}(\Omega X ; \mathbb{Z})$ is torsion-free.

Corollary 5.2. Let $K$ be a flag complex. Then $H_{*}\left(\Omega \mathcal{Z}_{K} ; \mathbb{Z}\right)$ is torsion-free.

Proof. Observe that $\Omega D J(\bar{K}) \simeq T^{m} \times \Omega \mathcal{Z}_{\bar{K}}$ and by Theorem 2.6, $\mathcal{Z}_{\bar{K}}$ is homotopy equivalent to a wedge of spheres. Thus $H_{*}(\Omega D J(\bar{K}))$ is torsion-free. By Theorem 4.1, $(\Omega D J(i))_{*}$ is onto for coefficients in any field. So by Lemma 5.1 . $H_{*}\left(\Omega \mathcal{Z}_{K} ; \mathbb{Z}\right)$ is torsion-free.

We now show that $\Omega \mathcal{Z}_{K}$ for $K$ flag is homotopy equivalent to a product of spheres and loops on spheres, when localised rationally or at any prime $p \neq 2$.

Theorem 5.3. Let $K$ be a flag complex. The following hold when localised rationally or at any prime $p \neq 2$ :

(a) the map $\Omega D J(\bar{K}) \stackrel{\Omega D J(i)}{\longrightarrow} \Omega D J(K)$ has a right homotopy inverse;

(b) the map $\Omega \mathcal{Z}_{\bar{K}} \stackrel{\Omega \mathcal{Z}(i)}{\longrightarrow} \Omega \mathcal{Z}_{K}$ has a right homotopy inverse;

(c) $\Omega D J(K)$ and $\Omega \mathcal{Z}_{K}$ are homotopy equivalent to products of spheres and loops on spheres.

Remark. Theorem 5.3 may be true integrally. Corollary [5.2 says there are no obstructions arising from torsion homology classes. When $K$ is Golod, so $\mathcal{Z}_{K}$ is homotopy equivalent to a wedge of spheres, then the integral statement is a consequence of the Hilton-Milnor Theorem. When $K$ is minimally non-Golod, so $\mathcal{Z}_{K}$ is homeomorphic to a connected sum of sphere products, then the integral statement holds by [3]. The methods in [3] arise in a different context and may or may not adapt to the case of $\mathcal{Z}_{K}$ for a general flag complex; at present not enough information is known about $\mathcal{Z}_{K}$. The methods presented below may possibly be fine-tuned to prove the integral case, but more delicate information would have to be known about the commutators in $H_{*}\left(\Omega \mathcal{Z}_{K}\right)$. In particular, Theorem 4.3 gives a minimal multiplicative basis for $H_{*}\left(\Omega \mathcal{Z}_{K}\right)$, but we do not know enough about potential relations among them.

Proof. We begin with an integral argument to establish some equivalences between statements in the theorem. After looping (5.1), we obtain a homotopy pullback diagram

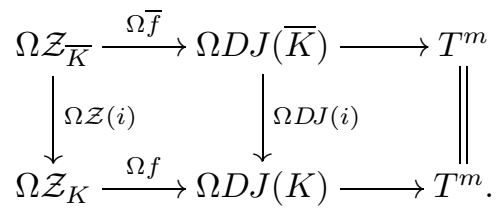


Since the fibration along the top row splits, it induces a splitting of the fibration along the bottom row. Therefore, using the loop structures in $\Omega D J(\bar{K})$ and $\Omega D J(K)$ to multiply, we obtain a homotopy commutative diagram of homotopy equivalences

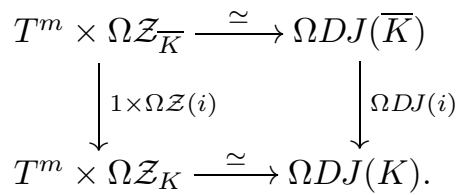

Thus $\Omega D J(i)$ has a right homotopy inverse if and only if $\Omega \mathcal{Z}(i)$ has a right homotopy inverse. Further, as $\Omega D J(K) \simeq T^{m} \times \Omega \mathcal{Z}_{K}$, we see that $\Omega D J(K)$ is homotopy equivalent to a product of spheres and loops on spheres if and only if $\Omega \mathcal{Z}_{K}$ is.

Now localise rationally or at a prime $p \neq 2$. It remains to show that $\Omega D J(i)$ has a right homotopy inverse and $\Omega D J(K)$ is homotopy equivalent to a product of spheres and loops on spheres. By Theorem 4.1, there are isomorphisms

$$
\begin{aligned}
& H_{*}(\Omega D J(\bar{K}) ; \mathbf{k}) \cong T\left\langle u_{1}, \ldots, u_{m}\right\rangle /\left(u_{i}^{2}=0\right), \\
& H_{*}(\Omega D J(K) ; \mathbf{k}) \cong T\left\langle u_{1}, \ldots, u_{m}\right\rangle /\left(u_{i}^{2}=0, u_{i} u_{j}+u_{j} u_{i}=0 \text { for }\{i, j\} \in K\right),
\end{aligned}
$$

where $\mathbf{k}$ is $\mathbb{Q}$ if we are localised rationally or $\mathbb{Z} / p \mathbb{Z}$ if localised at $p$. The free tensor algebra $T\left\langle u_{1}, \ldots, u_{m}\right\rangle$ is isomorphic to $U L\left\langle u_{1}, \ldots, u_{m}\right\rangle$, the universal enveloping algebra of the free Lie algebra on $u_{1}, \ldots, u_{m}$. The relations in the two tensor algebras above are induced from relations imposed on the underlying free Lie algebra $L\left\langle u_{1}, \ldots, u_{m}\right\rangle$. For as 2 is inverted in $\mathbf{k}$, the relation $u_{i}^{2}=0$ is equivalent to the relation $\left[u_{i}, u_{i}\right]=0$, and as each $u_{i}$ is of degree one, we have $u_{i} u_{j}+u_{j} u_{i}=\left[u_{i}, u_{j}\right]$. Thus there are isomorphisms

$$
\begin{aligned}
& T\left\langle u_{1}, \ldots, u_{m}\right\rangle /\left(u_{i}^{2}=0\right) \cong U\left(L\left\langle u_{1}, \ldots, u_{m}\right\rangle /\left(\left[u_{i}, u_{i}\right]=0\right)\right), \\
& T\left\langle u_{1}, \ldots, u_{m}\right\rangle /\left(u_{i}^{2}=0, u_{i} u_{j}+u_{j} u_{i}=0 \text { for }\{i, j\} \in K\right) \\
& \quad \cong U\left(L\left\langle u_{1}, \ldots, u_{m}\right\rangle /\left(\left[u_{i}, u_{i}\right]=0,\left[u_{i}, u_{j}\right]=0 \text { for }\{i, j\} \in K\right)\right) .
\end{aligned}
$$

To simplify notation, let

$$
\begin{aligned}
& \bar{L}=L\left\langle u_{1}, \ldots, u_{m}\right\rangle /\left(\left[u_{i}, u_{i}\right]=0\right), \\
& L=L\left\langle u_{1}, \ldots, u_{m}\right\rangle /\left(\left[u_{i}, u_{i}\right]=0,\left[u_{i}, u_{j}\right]=0 \text { for }\{i, j\} \in K\right) .
\end{aligned}
$$

Observe as well that in passing from loop space homology to universal enveloping algebras of Lie algebras, the map $(\Omega D J(i))_{*}$ is modelled by $U(\pi)$, where

$$
\pi: \bar{L} \longrightarrow L
$$

is the quotient map of Lie algebras. As a map of $\mathbf{k}$-modules, $\pi$ has a right inverse. Thus if $\widetilde{L}$ is the kernel of $\pi$, then by [9] there is an isomorphism of left $U \widetilde{L}$-modules

$$
U \bar{L} \cong U \widetilde{L} \otimes U L .
$$

Taking associated graded modules if necessary, by the Poincaré-Birkhoff-Witt Theorem we obtain an isomorphism of $\mathbf{k}$-modules

$$
S(\bar{L}) \cong S(\widetilde{L}) \otimes S(L)
$$

where $S($ ) is the free symmetric algebra functor.

In this case the Poincaré-Birkhoff-Witt Theorem has a geometric realisation. Since $\bar{K}$ is a disjoint union of points, by Theorem 2.6, there is an integral homotopy 
equivalence $\mathcal{Z}_{\bar{K}} \simeq \bigvee_{\ell=2}^{m}\left(S^{\ell+1}\right)^{\vee(\ell-1)}\left(\begin{array}{c}m \\ \ell\end{array}\right)$. Therefore there are integral homotopy equivalences

$$
\Omega D J(\bar{K}) \simeq T^{m} \times \Omega \mathcal{Z}_{\bar{K}} \simeq T^{m} \times \Omega\left(\bigvee_{\ell=2}^{m}\left(S^{\ell+1}\right)^{\vee(\ell-1)}\left(\begin{array}{c}
m \\
\ell
\end{array}\right)\right)
$$

The Hilton-Milnor Theorem gives an explicit decomposition of the loops on a wedge of spheres as an infinite product of looped spheres. In our case, we obtain an integral homotopy equivalence

$$
\Omega D J(\bar{K}) \simeq T^{m} \times \prod_{\alpha \in \mathcal{I}} \Omega S_{\alpha}
$$

for some index set $\mathcal{I}$, where each $S_{\alpha}$ is a sphere.

Take homology in (5.2) with $\mathbf{k}$ coefficients. We have $H_{*}\left(T^{m}\right) \cong \Lambda\left[u_{1}, \ldots, u_{m}\right]$, where each $u_{i}$ is of degree one. That is, $H_{*}\left(T^{m}\right) \cong \bigotimes_{i=1}^{m} S\left(u_{i}\right)$. Next, if the dimension of $S_{\alpha}$ is odd, say $S_{\alpha}=S^{2 k+1}$, then $H_{*}\left(\Omega S_{\alpha}\right) \cong \mathbf{k}\left[u_{\alpha}\right]$, where $\left|u_{\alpha}\right|=2 k$, so $H_{*}\left(\Omega S_{\alpha}\right) \cong S\left(u_{\alpha}\right)$. If the dimension of $S_{\alpha}$ is even, say $S_{\alpha}=S^{2 k}$, then the k-local splitting $\Omega S^{2 k} \simeq S^{2 k-1} \times \Omega S^{4 k-1}$ implies that $H_{*}\left(\Omega S_{\alpha}\right) \cong \Lambda\left[u_{\alpha}\right] \otimes \mathbf{k}\left[v_{\alpha}\right]$, where $\left|u_{\alpha}\right|=2 k-1$ and $\left|v_{\alpha}\right|=4 k-1$, so $H_{*}\left(\Omega S_{\alpha}\right) \cong S\left(u_{\alpha}\right) \otimes S\left(v_{\alpha}\right)$. Putting all this together, (5.2) implies that there is a coalgebra isomorphism

$$
H_{*}(\Omega D J(\bar{K}) ; \mathbf{k}) \cong \bigotimes_{\alpha^{\prime} \in \mathcal{I}^{\prime}} S\left(u_{\alpha^{\prime}}\right)
$$

where the index set $\mathcal{I}^{\prime}$ consists of $\{1,2, \ldots, m\}$, every $\alpha \in \mathcal{I}$ where $S_{\alpha}$ is of odd dimension, and two indices $\alpha_{2 k-1}, \alpha_{4 k-1}$ for every $\alpha \in \mathcal{I}$ where $S_{\alpha}$ is of dimension $2 k$.

We now have two descriptions of $H_{*}(\Omega D J(\bar{K}))$ as symmetric algebras, so there is an isomorphism

$$
S(\bar{L}) \cong \bigotimes_{\alpha^{\prime} \in \mathcal{I}^{\prime}} S\left(u_{\alpha^{\prime}}\right)
$$

On the other hand, there is a decomposition $S(\bar{L}) \cong S(\widetilde{L}) \otimes S(L)$, so we can choose a new index set $\mathcal{J} \subseteq \mathcal{I}^{\prime}$ such that the composite

$$
\bigotimes_{\beta \in \mathcal{J}} S\left(u_{\beta}\right) \hookrightarrow \bigotimes_{\alpha^{\prime} \in \mathcal{I}^{\prime}} S\left(u_{\alpha^{\prime}}\right) \stackrel{\cong}{\longrightarrow} S(\bar{L}) \stackrel{\text { proj }}{\longrightarrow} S(L)
$$

is an isomorphism. Write $\mathcal{J}=\mathcal{J}_{1} \sqcup \mathcal{J}_{2}$ where $\mathcal{J}_{1}$ (respectively $\mathcal{J}_{2}$ ) consists of all those $\beta \in \mathcal{J}$ with $\left|u_{\beta}\right|$ odd (respectively even). Observe that (5.3) is induced in homology by the composite

$$
\left(\prod_{\beta \in \mathcal{J}_{1}} S_{\beta}\right) \times\left(\prod_{\beta \in \mathcal{J}_{2}} \Omega S_{\beta}\right) \hookrightarrow T^{m} \times \prod_{\alpha \in \mathcal{I}} \Omega S_{\alpha} \stackrel{\simeq}{\longrightarrow} \Omega D J(\bar{K}) \stackrel{\Omega D J(i)}{\longrightarrow} \Omega D J(K) .
$$

The left map exists k-locally, since there is a $\mathbf{k}$-local decomposition $\Omega S^{2 k} \simeq S^{2 k-1} \times$ $\Omega S^{4 k-1}$. Thus if we take $\varphi$ to be the composite of the left and middle maps above, then $\varphi$ has property that $\Omega D J(i) \circ \varphi$ induces an isomorphism in $\mathbf{k}$-homology. This completes the proof. 


\section{An example: The boundary of a pentagon}

In this section we consider an example which illustrates many of the ideas and results of the paper. This is most fully discussed once the algebra generators of $H_{*}(\Omega D J(K))$ are geometrically realised by Samelson products, so we begin with a general lemma.

Let $K$ be a flag complex which is Golod. As in Section 5 , let $\bar{K}$ be the disjoint union of the $m$ vertices in $K$. We obtain an inclusion $i: \bar{K} \longrightarrow K$ which induces an inclusion $D J(i): D J(\bar{K})=\bigvee_{j=1}^{m} \mathbb{C} P^{\infty} \longrightarrow D J(K)$. For $1 \leqslant i \leqslant m$, let $\bar{\mu}_{i}$ be the composite

$$
\bar{\mu}_{i}: S^{2} \longrightarrow \mathbb{C} P^{\infty} \longrightarrow \bigvee_{j=1}^{m} \mathbb{C} P^{\infty} \stackrel{\operatorname{DJ(i)}}{\longrightarrow} \operatorname{DJ}(K)
$$

where the left map is the inclusion of the bottom cell and the middle map is the inclusion of the $i^{t h}$-wedge summand. Let

$$
\mu_{i}: S^{1} \longrightarrow \Omega D J(K)
$$

be the adjoint of $\bar{\mu}_{i}$. Then in the description of $H_{*}(\Omega D J(K))$ in (4.1), the Hurewicz image of $\mu_{i}$ is the algebra generator $u_{i}$.

Since the Samelson product commutes with the Hurewicz homomorphism, the Hurewicz image of any iterated Samelson product of the $\mu_{i}$ 's is the corresponding iterated commutator of the $u_{i}$ 's. As well, in the homotopy fibration $\Omega \mathcal{Z}_{K} \longrightarrow$ $\Omega D J(K) \longrightarrow T^{m}$, since $\pi_{k}\left(T^{m}\right)=0$ for $k>1$, any iterated Samelson product of the $\mu_{i}$ 's composes trivially into $T^{m}$ and so lifts to $\Omega \mathcal{Z}_{K}$.

Since we are regarding $H_{*}\left(\Omega \mathcal{Z}_{K}\right)$ as the commutator subalgebra of $H_{*}(\Omega D J(K))$ via exact sequence (2.1), we can regard the lift to $\Omega \mathcal{Z}_{K}$ of any iterated Samelson product of the $\mu_{i}$ 's as having the same Hurewicz image. Therefore, the algebra generators

$$
\left[u_{j}, u_{i}\right], \quad\left[u_{k_{1}},\left[u_{j}, u_{i}\right]\right], \quad \cdots, \quad\left[u_{k_{1}},\left[u_{k_{2}}, \cdots\left[u_{k_{m-2}},\left[u_{j}, u_{i}\right]\right] \cdots\right]\right]
$$

of $H_{*}\left(\Omega \mathcal{Z}_{K}\right)$ in Theorem 4.3, with restrictions on the indices as stated in the theorem, are the Hurewicz images of the lifts to $\Omega \mathcal{Z}_{K}$ of the iterated Samelson products

$$
\left[\mu_{j}, \mu_{i}\right], \quad\left[\mu_{k_{1}},\left[\mu_{j}, \mu_{i}\right]\right], \quad \ldots, \quad\left[\mu_{k_{1}},\left[\mu_{k_{2}}, \cdots\left[\mu_{k_{m-2}},\left[\mu_{j}, \mu_{i}\right]\right] \cdots\right]\right] .
$$

Lemma 6.1. Let $K$ be a flag complex and $\mathbf{k}$ a field. Suppose that $K$ is Golod, or equivalently by Theorem 4.6, that $\mathcal{Z}_{K}$ is homotopy equivalent to a wedge of spheres. Then each sphere in this wedge maps to DJ $K)$ by an iterated Whitehead product of the maps $\bar{\mu}_{1}, \ldots, \bar{\mu}_{m}$.

Proof. Since $\mathcal{Z}_{K}$ is homotopy equivalent to a wedge of spheres, $H_{*}\left(\Omega \mathcal{Z}_{K}\right)$ is a free associative algebra, where each algebra generator of degree $d$ corresponds to a sphere of dimension $d+1$ in the wedge decomposition of $\mathcal{Z}_{K}$. On the other hand, a minimal generating set for $H_{*}\left(\Omega \mathcal{Z}_{K}\right)$ is given by the iterated commutators in Theorem 4.3 , so each iterated commutator listed in Theorem 4.3 of degree $d$ corresponds to a sphere of dimension $d+1$ in the wedge decomposition of $\mathcal{Z}_{K}$. Applying the map $\Omega \mathcal{Z}_{K} \longrightarrow \Omega D J(K)$, these iterated commutators are the Hurewicz images of the iterated Samelson products in (6.1). Therefore, adjointing, the spheres in the wedge decomposition of $\mathcal{Z}_{K}$ map to $D J(K)$ by the iterated Whitehead products

$$
\left[\bar{\mu}_{j}, \bar{\mu}_{i}\right], \quad\left[\bar{\mu}_{k_{1}},\left[\bar{\mu}_{j}, \bar{\mu}_{i}\right]\right], \quad \ldots, \quad\left[\bar{\mu}_{k_{1}},\left[\bar{\mu}_{k_{2}}, \cdots\left[\bar{\mu}_{k_{m-2}},\left[\bar{\mu}_{j}, \bar{\mu}_{i}\right]\right] \cdots\right]\right]
$$

with restrictions on the indices as in Theorem 4.3 . 


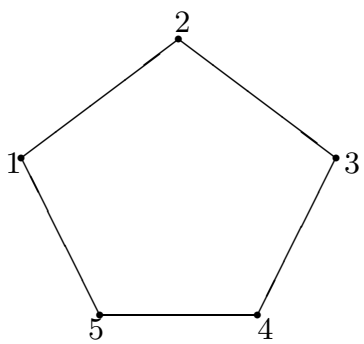

FiguRE 2. Boundary of a pentagon.

Now let $K$ be the boundary of a pentagon, shown in Figure 2 Theorem 4.3 gives the following ten generators for the Pontryagin algebra $H_{*}\left(\Omega \mathcal{Z}_{K}\right)$ :

$$
\begin{gathered}
a_{1}=\left[u_{3}, u_{1}\right], \quad a_{2}=\left[u_{4}, u_{1}\right], \quad a_{3}=\left[u_{4}, u_{2}\right], \quad a_{4}=\left[u_{5}, u_{2}\right], \quad a_{5}=\left[u_{5}, u_{3}\right], \\
b_{1}=\left[u_{4},\left[u_{5}, u_{2}\right]\right], \quad b_{2}=\left[u_{3},\left[u_{5}, u_{2}\right]\right], \quad b_{3}=\left[u_{1},\left[u_{5}, u_{3}\right]\right], \\
b_{4}=\left[u_{3},\left[u_{4}, u_{1}\right]\right], \quad b_{5}=\left[u_{2},\left[u_{4}, u_{1}\right]\right],
\end{gathered}
$$

where $\operatorname{deg} a_{i}=2$ and $\operatorname{deg} b_{i}=3$. By Lemma 6.1, $a_{1}$ is the Hurewicz image of the Samelson product $\left[\mu_{3}, \mu_{1}\right]: S^{2} \rightarrow \Omega D J(K)$ lifted to $\Omega \mathcal{Z}_{K}$, and $b_{1}$ is the Hurewicz image of the iterated Samelson product $\left[\mu_{4},\left[\mu_{5}, \mu_{2}\right]\right]: S^{3} \rightarrow \Omega D J(K)$ lifted to $\Omega \mathcal{Z}_{K}$; the other $a_{i}$ and $b_{i}$ are described similarly. We therefore have adjoint maps

$$
\epsilon:\left(S^{2} \vee S^{3}\right)^{\vee 5} \rightarrow \Omega \mathcal{Z}_{K} \quad \text { and } \quad j:\left(S^{3} \vee S^{4}\right)^{\vee 5} \rightarrow \mathcal{Z}_{K}
$$

corresponding to the wedge of all $a_{i}$ and $b_{i}$. Now a calculation using relations from Theorem 4.1 and the Jacobi identity shows that $a_{i}$ and $b_{i}$ satisfy the relation

$$
\sum_{i=1}^{5}\left[a_{i}, b_{i}\right]=0
$$

(the signs can be made right by changing the order of the elements in the commutators defining $a_{i}, b_{i}$ if necessary). This relation has a topological meaning. In general, suppose that $M$ and $N$ are $d$-dimensional manifolds. Let $\bar{M}$ be the $(d-1)$ skeleton of $M$, or equivalently, $\bar{M}$ is obtained from $M$ by removing a disc in the interior of the $d$-cell of $M$. Define $\bar{N}$ similarly. Suppose that $f: S^{d-1} \longrightarrow \bar{M}$ and $g: S^{d-1} \longrightarrow \bar{N}$ are the attaching maps for the top cells in $M$ and $N$. Then the attaching map for the top cell in the connected sum $M \# N$ is $S^{d-1} \stackrel{f+g}{\longrightarrow} \bar{M} \vee \bar{N}$. In our case, $S^{3} \times S^{4}$ is a manifold and the attaching map $S^{6} \longrightarrow S^{3} \vee S^{4}$ for its top cell is the Whitehead product $\left[s_{1}, s_{2}\right]$, where $s_{1}$ and $s_{2}$ respectively are the inclusions of $S^{3}$ and $S^{4}$ into $S^{3} \vee S^{4}$. The attaching map for the top cell of the 5-fold connected sum $\left(S^{3} \times S^{4}\right)^{\# 5}$ is therefore the sum of five such Whitehead products. Composing it with $j$ into $\mathcal{Z}_{K}$ and passing to the adjoint map we obtain $\sum_{i=1}^{5}\left[a_{i}, b_{i}\right]$. By (6.2), this sum is null homotopic. Thus the inclusion $j:\left(S^{3} \vee S^{4}\right)^{\vee 5} \rightarrow \mathcal{Z}_{K}$ extends to a map

$$
\widetilde{j}:\left(S^{3} \times S^{4}\right)^{\# 5} \rightarrow \mathcal{Z}_{K}
$$

Furthermore, a calculation using Theorem 2.5$]$ shows that $\widetilde{j}$ induces an isomorphism in cohomology (see [7, Ex. 7.22]), that is, $\widetilde{j}$ is a homotopy equivalence. Since both $\left(S^{3} \times S^{4}\right)^{\# 5}$ and $\mathcal{Z}_{K}$ are manifolds, the complement of $\left(S^{3} \vee S^{4}\right)^{\vee 5}$ in $\left(S^{3} \times S^{4}\right)^{\# 5}$ 
and $\mathcal{Z}_{K}$ is a 7 -disc, so that the extension map $\widetilde{j}$ can be chosen to be one-to-one, which implies that $\widetilde{j}$ is a homeomorphism.

We also obtain that $H_{*}\left(\Omega \mathcal{Z}_{K}\right)$ is the quotient of a free algebra on ten generators $a_{i}, b_{i}$ by relation (6.2). Its Poincaré series is given by Proposition 4.2

$$
P\left(H_{*}\left(\Omega \mathcal{Z}_{K}\right) ; t\right)=\frac{1}{1-5 t^{2}-5 t^{3}+t^{5}} .
$$

The summand $t^{5}$ in the denominator is what differs the Poincaré series of the onerelator algebra $H_{*}\left(\Omega \mathcal{Z}_{K}\right)$ from that of the free algebra $H_{*}\left(\Omega\left(S^{3} \vee S^{4}\right)^{\vee 5}\right)$.

A similar argument can be used to show that $\mathcal{Z}_{K}$ is homeomorphic to a connected sum of sphere products when $K$ is a boundary of an $m$-gon with $m \geqslant 4$.

\section{ACKNOWLEDGEMENT}

The authors would like to thank the International Centre for Mathematical Sciences in Edinburgh, whose support through a Research-in-Groups grant allowed the authors to work together on these problems for a month in Aberdeen.

\section{REFERENCES}

[1] A. Bahri, M. Bendersky, F. R. Cohen, and S. Gitler, The polyhedral product functor: a method of decomposition for moment-angle complexes, arrangements and related spaces, Adv. Math. 225 (2010), no. 3, 1634-1668, DOI 10.1016/j.aim.2010.03.026. MR2673742(2012b:13053)

[2] I. V. Baskakov, V. M. Bukhshtaber, and T. E. Panov, Algebras of cellular cochains, and torus actions (Russian), Uspekhi Mat. Nauk 59 (2004), no. 3(357), 159-160, DOI 10.1070/RM2004v059n03ABEH000743; English transl., Russian Math. Surveys 59 (2004), no. 3, 562-563. MR2117435(2006b:57045)

[3] Piotr Beben and Stephen Theriault, The loop space homotopy type of simply-connected four-manifolds and their generalizations, Adv. Math. 262 (2014), 213-238, DOI 10.1016/j.aim.2014.05.015. MR.3228428

[4] Alexander Berglund and Michael Jöllenbeck, On the Golod property of Stanley-Reisner rings, J. Algebra 315 (2007), no. 1, 249-273, DOI 10.1016/j.jalgebra.2007.04.018. MR.2344344 (2008f:13036)

[5] Frédéric Bosio and Laurent Meersseman, Real quadrics in $\mathbf{C}^{n}$, complex manifolds and convex polytopes, Acta Math. 197 (2006), no. 1, 53-127, DOI 10.1007/s11511-006-0008-2. MR2285318 (2007j:32037)

[6] Victor M. Buchstaber and Taras E. Panov, Torus actions determined by simple polytopes, Geometry and topology: Aarhus (1998), Contemp. Math., vol. 258, Amer. Math. Soc., Providence, RI, 2000, pp. 33-46, DOI 10.1090/conm/258/04054. MR.1778095 (2001h:57046)

[7] Victor M. Buchstaber and Taras E. Panov, Torus actions and their applications in topology and combinatorics, University Lecture Series, vol. 24, American Mathematical Society, Providence, RI, 2002. MR1897064 (2003e:57039)

[8] Victor M. Buchstaber and Taras E. Panov, Toric Topology, arXiv:1210.2368.

[9] F. R. Cohen, J. C. Moore, and J. A. Neisendorfer, Torsion in homotopy groups, Ann. of Math. (2) 109 (1979), no. 1, 121-168, DOI 10.2307/1971269. MR.519355 (80e:55024)

[10] F. R. Cohen and J. A. Neisendorfer, A construction of p-local H-spaces, Algebraic topology, Aarhus 1982 (Aarhus, 1982), Lecture Notes in Math., vol. 1051, Springer, Berlin, 1984, pp. 351-359, DOI 10.1007/BFb0075576. MR764588 (86e:55011)

[11] Michael W. Davis and Tadeusz Januszkiewicz, Convex polytopes, Coxeter orbifolds and torus actions, Duke Math. J. 62 (1991), no. 2, 417-451, DOI 10.1215/S0012-7094-91-06217-4. MR.1104531 (92i:52012)

[12] M. Franz, The integral cohomology of toric manifolds, Tr. Mat. Inst. Steklova 252 (2006), no. Geom. Topol., Diskret. Geom. i Teor. Mnozh., 61-70; English transl., Proc. Steklov Inst. Math. 1 (252) (2006), 53-62. MR2255969(2007f:14050)

[13] Ralph Fröberg, Determination of a class of Poincaré series, Math. Scand. 37 (1975), no. 1, 29-39. MR0404254 (53 \#8057) 
[14] D. R. Fulkerson and O. A. Gross, Incidence matrices and interval graphs, Pacific J. Math. 15 (1965), 835-855. MR0186421 (32 \#3881)

[15] Jelena Grbić and Stephen Theriault, Homotopy type of the complement of a configuration of coordinate subspaces of codimension two (Russian), Uspekhi Mat. Nauk 59 (2004), no. 6(360), 203-204, DOI 10.1070/RM2004v059n06ABEH000803; English transl., Russian Math. Surveys 59 (2004), no. 6, 1207-1209. MR2138475 (2005k:55023)

[16] Jelena Grbić and Stephen Theriault, The homotopy type of the complement of a coordinate subspace arrangement, Topology 46 (2007), no. 4, 357-396, DOI 10.1016/j.top.2007.02.006. MR2321037 (2008j:13051)

[17] Jelena Grbić and Stephen Theriault, The homotopy type of the polyhedral product for shifted complexes, Adv. Math. 245 (2013), 690-715, DOI 10.1016/j.aim.2013.05.002. MR.3084441

[18] Evgeniy S. Golod, On the cohomology of some local rings (Russian), Soviet Math. Dokl. 3 (1962), 745-749.

[19] Tor H. Gulliksen and Gerson Levin, Homology of local rings, Queen's Paper in Pure and Applied Mathematics, No. 20, Queen's University, Kingston, Ont., 1969. MR0262227(41 \#6837)

[20] J. Herzog, V. Reiner, and V. Welker, Componentwise linear ideals and Golod rings, Michigan Math. J. 46 (1999), no. 2, 211-223, DOI 10.1307/mmj/1030132406. MR.1704158 (2000d:13032)

[21] Taras E. Panov and Nigel Ray, Categorical aspects of toric topology, Toric topology, Contemp. Math., vol. 460, Amer. Math. Soc., Providence, RI, 2008, pp. 293-322, DOI 10.1090/conm/460/09026. MR2428364(2009j:55026)

[22] Taras Panov, Nigel Ray, and Rainer Vogt, Colimits, Stanley-Reisner algebras, and loop spaces, Categorical decomposition techniques in algebraic topology (Isle of Skye, 2001), Progr. Math., vol. 215, Birkhäuser, Basel, 2004, pp. 261-291. MR2039770 (2004k:55008)

School of Mathematics, University of Southampton, Southampton SO17 1BJ, United KINGDOM

E-mail address: J.Grbic@soton.ac.uk

Department of Mathematics and Mechanics, Moscow State University, Leninskie Gory, 119991 Moscow, Russia - And - Institute for Theoretical and Experimental Physics, Moscow, Russia - And - Institute for Information Transmission Problems, RusSian ACADEMy of Sciences, Moscow, Russia

E-mail address: tpanov@mech.math.msu.su

School of Mathematics, University of Southampton, Southampton SO17 1BJ, United KINGDOM

E-mail address: S.D.Theriault@soton.ac.uk

Department of Mathematics, National University of Singapore, Block S17 (SOC1), 06-02 10, Lower Kent Ridge Road, 119076 Singapore

E-mail address: matwuj@nus.edu.sg 